\title{
Numerical analysis of the attitude stability of a charged spacecraft in the Pitch-Roll-Yaw directions
}

\author{
Yehia A. Abdel-Aziz* \\ National Research Institute of Astronomy and Geophysics (NRIAG), Cairo, Egypt \\ University of Hail, Department of Mathematics, PO BOX 2440, Kingdom of Saudi Arabia \\ Muhammad Shoaib** \\ University of Hail, Department of Mathematics, PO BOX 2440, Kingdom of Saudi Arabia
}

\begin{abstract}
In this paper, the effect of Lorentz force on the stability of attitude orientation of a charged spacecraft moving in an elliptic orbit in the geomagnetic field is considered. Euler equations are used to derive the equations of attitude motion of a charged spacecraft. The equilibrium positions and its stability are investigated separately in the pitch, roll and yaw directions. In each direction, we use the Lorentz force to identify an attitude stabilization parameter. The analytical methods confirm that we can use the Lorentz force as a stabilization method. The charge-to-mass ratio is the main key of control, in addition to the components of the radius vector of the charged center of the spacecraft, relative to the center of mass of the spacecraft. The numerical results determine stable and unstable equilibrium positions. Therefore, in order to generate optimum charge, which may stabilize the attitude motion of a spacecraft, the amount of charge on the surface of spacecraft will need to be monitored for passive control.
\end{abstract}

Key words: Lorentz force, Charged spacecraft, Attitude dynamics, Control of spacecraft, Stability

\section{Introduction}

An important aspect of the attitude dynamics of a spacecraft is the control of its attitude motion. Recently, a novel attitude orientation and formation flying concept using electrostatic propulsion has been proposed in [1], [2], and [3]. The charge of the spacecraft is controlled, to generate inter-spacecraft Coulomb forces. Such forces can be used to re-orient, or attract or repel the spacecraft from each other, and thus control their relative attitude motion. Studying the electrostatic charging data of the geostationary SCATHA spacecraft 7 , it became evident that it is possible to generate forces of the order of $10-1000 \mu N$ [4]. The phenomenon of spacecraft surface charging was discovered after the launch of an artificial satellite, and therefore the orbital motion of a charged artificial satellite affected by Lorentz force was studied by [5], [6] and [7]. Therefore, the Lorentz force is a possible means for charging, and thus controlling spacecraft orbits, without consuming propellant. The work in [8] was the first to introduce a control scheme using Lorentz augmented orbits. The spacecraft orbits accelerated by the Lorentz force are termed Lorentz-augmented orbits, because the Lorentz force cannot completely replace the traditional rocket propulsion. After [9], a series of papers [10-13] applied charged control techniques to the utilization of Lorentz forces for satellite orbit control.

In [14], the author studied the stability of the equilibrium position due to Lorentz torque, in the case of a uniform magnetic field, and cylindrical shape of an artificial satellite, moving in circular orbit. In [15], the author investigated the
This is an Open Access article distributed under the terms of the Creative Commons Attribution Non-Commercial License (http://creativecommons.org/licenses/by$\mathrm{nc} / 3.0 /$ ) which permits unrestricted non-commercial use, distribution, and reproduction in any medium, provided the original work is properly cited. (c) * Corresponding author : yehia@nriag.sci.eg ** safridi@gmail.com 
attitude motion of a charged pendulum satellite moving in circular orbit, having the shape of a dumbbell pendulum, due to Lorentz torque. Their studies of the stability of equilibrium points focused only on pitch position, within the equatorial plane.

In this paper, we develop a new model for the torque due to the Lorentz force, for the general shape of a spacecraft moving in an elliptic orbit, in the Earth's magnetic field. We assume that the the Earth's magnetic field is acting as a dipole. The main objective of this work is to study the possibility of using Lorentz force as a source of attitude stabilization. We developed components of the Lorentz torque, as functions of orbital elements. This allows us to study the relation between translation motion and rotational motion of the spacecraft, which is not possible in the case of [15], who considers the spacecraft moving in circular orbit only. The equations of motion for the attitude orientation are developed, using Euler-Poisson Equations. We investigate the equilibrium positions and its stability separately in the pitch, roll and yaw directions. Studying all the three directions is important, because it is not guaranteed that if the spacecraft is stable at an equilibrium in one direction, then it will be stable in another direction as well, for example the roll direction or yaw direction. In addition, sometimes it is also possible to achieve a maneuver in different directions, and therefore the one which consumes less energy will be chosen. Therefore, it is important to know stable equilibriums in all three directions. We also identify attitude stabilization parameters, and determine the parameter of passive control, using Lorentz force for each direction. The analytical solution confirms that the charge-to-mass ratio is the main key of stabilization, in addition to the components of the radius vector of the charge center of a spacecraft, relative to the center of mass of the spacecraft. Numerical analysis are is used to identify stable and unstable equilibrium positions.

\section{Spacecraft model and Torque due to Lo- rentz force}

\subsection{Spacecraft model}

A rigid spacecraft is considered, whose center of mass moves in the Newtonian central gravitational field of the earth, in an elliptic orbit. We suppose that the spacecraft is equipped with an electrostatic charged protective shield, having an intrinsic magnetic moment. The rotational motion of the spacecraft about its center of mass is analyzed, considering the influence of gravity gradient torque $T_{G}$, and the torque $T_{L}$ due to Lorentz forces, respectively. The torque
$T_{L}$ results from the interaction of the geomagnetic field with the charged screen of the electrostatic shield.

The rotational motion of the satellite relative to its center of mass is investigated in the orbital coordinate system $C_{x_{x} y_{0} z_{0}}$ with $C_{x_{0}}$ tangential to the orbit in the direction of motion, $C_{y_{0}}$ lying along the normal to the orbital plane, and $C_{z_{0}}$ lying along the radius vector $r$ of the point $O_{E}$, relative to the center of the Earth. The investigation is carried out assuming the rotation of the orbital coordinate system, relative to the inertial system, with the angular velocity $\Omega$. The system $O_{X Y Z}$ is taken as an inertial coordinate system, whose axis $O Z(k)$ is directed along the axis of the Earth's rotation, the axis $O X(i)$ is directed toward the ascending node of the orbit, and the plane coincides with the equatorial plane. Also, we assume that the satellite's principal axes of inertia $C_{x_{b} y_{b} z_{b}}$ are rigidly fixed to the satellite $\left(i_{b}, j_{b}, k_{b}\right)$. The satellite's attitude may be described in several ways; in this paper, the attitude will be described by the angle of yaw $\psi$, the angle of pitch $\theta$, and the angle of roll $\varphi$, between the axes $C_{x_{y_{b} y_{b}} z_{b}}$ and $O_{X Y Z}$. The three angles are obtained by rotating satellite axes from an attitude coinciding with the reference axes, to describe the attitude in the following way:

- The angle of precession $\psi$ is taken in the plane orthogonal to the $Z$-axis.

- $\theta$ is the notation angle between the axes $Z$ and $z_{0}$

- $\phi$ is the angle of self -rotation around the $Z$-axis

According to [16], we can write the relationship between the reference frames $C_{x_{b_{b} y_{b} z_{b}}}$ and $C_{x_{\alpha_{0} z_{0}}}$ as given by the matrix $\mathrm{A}$, which is the matrix of the direction cosines $\alpha_{i}, \beta_{i}, \gamma_{i},(i=1$, $2,3)$

$$
A=\left(\begin{array}{lll}
\alpha_{1} & \alpha_{2} & \alpha_{3} \\
\beta_{1} & \beta_{2} & \beta_{3} \\
\gamma_{1} & \gamma_{2} & \gamma_{3}
\end{array}\right),
$$

where,

$$
\begin{aligned}
& \alpha_{1}=\cos \psi \cos \phi-\sin \psi \sin \phi \cos \theta, \\
& \alpha_{2}=-\cos \psi \sin \phi-\cos \theta \sin \psi \cos \phi, \\
& \alpha_{3}=\sin \theta \sin \psi, \\
& \beta_{1}=\sin \psi \cos \varphi+\cos \theta \cos \psi \sin \varphi, \\
& \beta_{2}=-\sin \psi \sin \phi+\cos \theta \cos \psi \cos \phi, \\
& \left.\beta_{3}=-\sin \theta \cos \psi\right), \\
& \gamma_{1}=\sin \theta \sin \phi, \\
& \gamma_{2}=\sin \theta \cos \phi, \\
& \gamma_{3}=\cos \theta,
\end{aligned}
$$

and,

$$
\vec{\alpha}=\alpha_{1} i_{b}+\alpha_{2} j_{b}+\alpha_{3} k_{b}, \vec{\beta}=\beta_{1} i_{b}+\beta_{2} j_{b}+\beta_{3} k_{b}, \vec{\gamma}=\gamma_{1} i_{b}+\gamma_{2} j_{b}+\gamma_{3} k_{b},
$$




\section{Torque due to Lorentz Force}

We use spherical coordinates to describe the magnetic and gravitational fields, and the spacecraft trajectory, as shown in Figure 1; and the $x, y$ and $z$ axes form a set of Inertial Cartesian coordinates. The Earth is assumed to rotate about the $z$-axes. The magnetic dipole is not tilted, and is therefore, axi-symmetric. The spherical coordinates consist of radius $r$, colatitude angle $\Phi$, and azimuth from the $x$ direction $\Theta$ (see Figure 1).

The magnetic field is expressed as

$$
\vec{B}=\frac{B_{0}}{r^{3}}[2 \cos \Phi \hat{r}+\sin \Phi \hat{\Phi}+\overrightarrow{0} \hat{\Theta}]
$$

where, $B_{0}$ is the strength of the magnetic field in Wb.m. The acceleration in inertial coordinates is given by

$$
\vec{a}=\frac{\vec{F}}{m}=-\frac{\mu}{r^{3}} \vec{r}+\frac{q}{m}\left(\vec{V}_{r e l} \times \vec{B}\right),
$$

where, $\frac{q}{m}$ is the charge-to-mass ration of the spacecraft, and $\vec{V}_{r e l}$ is the velocity of the spacecraft relative to the magnetic field of the Earth. The Lorentz force (per unit mass) can be written as follows:

$$
\begin{aligned}
& \vec{F}_{L}=\frac{q}{m}\left(\vec{V}_{r e l} \times \vec{B}\right), \\
& \vec{V}_{r e l}=\vec{V}-\vec{\omega}_{e} \times \vec{r},
\end{aligned}
$$

where, $\vec{V}$ is the inertial velocity of the spacecraft, and $\vec{\omega}_{e}$ is the angular velocity vector of the Earth. According to [11], we use

$$
\vec{V}=\dot{r} \hat{r}+r \dot{\Phi} \hat{\Phi}+r \dot{\Theta} \sin \Phi \hat{\Theta}
$$

and,

$$
\vec{r}=r \hat{r}
$$

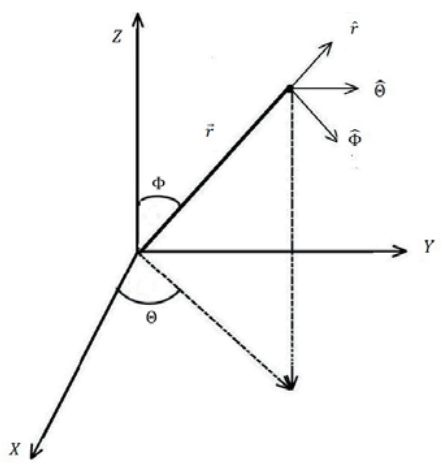

Fig. 1. Spherical coordinates used in the derivation of the equations of motion

$$
\begin{aligned}
& \vec{\omega}_{e}=\omega_{e} \hat{z}, \\
& \vec{z}=\cos \Phi \hat{r}-\sin \Phi \hat{\Phi} .
\end{aligned}
$$

Therefore, the acceleration in inertial coordinates is given by:

$$
\vec{F}_{L}=\frac{q B_{0}}{m r^{2}}\left[-\left(\dot{\Theta}-\omega_{e}\right)\left(\sin ^{2} \Phi \hat{r}+\sin (2 \Phi) \hat{\Phi}\right)+\left(\frac{\dot{r}}{r} \sin \Phi-2 \dot{\Phi} \cos \Phi\right) \hat{\Theta}\right]
$$

In the case of Torque, it is very important that $\vec{F}_{L}$ is decomposed into radial, transverse, and normal components $\left(R_{L}, T_{L}, N_{L}\right)$. The unit vector $\hat{n}$ normal to the orbit is collinear with the angular momentum unit vector $\hat{h}$.

$$
\hat{n}=\hat{h}=(\vec{r} \times \vec{V}) / \sqrt{\mu p}=r^{2} / \sqrt{\mu p}(-\dot{\Theta} \sin \Phi \hat{\Phi}+\dot{\Phi} \hat{\Theta}),
$$

where, $p=a\left(1-e^{2}\right), \mu$ is the Earth's gravitational parameter, $a$ is the semi-major axis, and $e$ is the eccentricity of the satellite's orbit. The transverse unit vector $\hat{t}$ can be calculated from the right-handed set, $\hat{t}=\hat{n} \times \hat{r}$. Decomposition of the Lorentz force experienced by the geomagnetic field into the radial, transverse, and normal components, respectively, yields:

$$
\begin{aligned}
& R_{L}=\vec{F}_{L} \cdot \hat{r}=\frac{q}{m} \frac{B_{0}}{r^{2}}\left[\omega_{e}-\dot{\Theta}\right] \sin ^{2} \Phi \\
& T_{L}=\vec{F}_{L} \cdot \hat{t}=\frac{q}{m} \frac{B_{0}}{\sqrt{\mu p}}\left[\frac{\dot{r}}{r} \dot{\Theta} \sin ^{2} \Phi-2 \omega_{e} \dot{\Phi} \cos \Phi \sin \Phi\right] \\
& N_{L}=\vec{F}_{L} \cdot \hat{n}=\frac{q}{m} \frac{B_{0}}{\sqrt{\mu p}}\left[2 \dot{\Theta}\left(\omega_{e}-\dot{\Theta}\right) \sin ^{2} \Phi \cos \Phi+\frac{\dot{r}}{r} \dot{\Theta} \sin \Phi-2 \dot{\Phi}^{2} \cos \Phi\right] .
\end{aligned}
$$

The relationship between the spherical coordinates and the orbital elements is required, to write the components of the Lorentz force as a function of the orbital elements.

$$
\begin{aligned}
& r=p /(1+e \cos f), \\
& \dot{r}=e \sqrt{\mu / p} \sin f, \\
& \cos \Phi=\sin i \sin (\omega+f), \\
& \sin \Phi=\sqrt{1-\sin ^{2} i \sin ^{2}(\omega+f),} \\
& \dot{\Phi}=-\sqrt{\mu / p^{3}} \frac{\sin i \cos (\omega+f)}{\sqrt{1-\sin ^{2} i \cos ^{2}(\omega+f)}}(1+e \cos f)^{2}, \\
& \dot{\Theta}=\sqrt{\mu / p^{3}} \frac{\cos ^{2}}{1-\sin ^{2} i \sin ^{2}(\omega+f)}(1+e \cos f)^{2},
\end{aligned}
$$

where, $i, \omega$ and $f$ are the inclination of the orbit on the equator, argument of the perigee, and the true anomaly of the spacecraft orbit, respectively. Therefore, rewriting the 
components of the Lorentz force due to the geomagnetic field as a function of the orbital elements, we obtain

$$
\begin{aligned}
& R_{L}=\frac{q}{m} \frac{B_{0}}{r^{2}}\left[\omega_{e}\left[1-\sin ^{2} i \sin ^{2}(\omega+f)\right]-\sqrt{\mu / p^{3}} \cos i(1+e \cos f)^{2}\right], \\
& T_{L}=\frac{q}{m} \frac{B_{0}}{\sqrt{\mu p}}\left[\begin{array}{l}
\dot{r} \\
r \\
\sin ^{2} i \sin (\omega+f) \cos (\omega+f)(1+e \cos f)^{2}
\end{array}\right], \\
& N_{L}=\frac{q}{m} \frac{B_{0}}{\sqrt{\mu p}}\left[\begin{array}{c}
2\left[\omega_{e}\left[1-\sin ^{2} i \sin ^{2}(\omega+f)\right]-\sqrt{\mu / p^{3}} \cos i(1+e \cos f)^{2}\right] \times \\
\sqrt{\mu / p^{3}} \cos i(1+e \cos f)^{2}+\frac{\dot{r}}{r} \sqrt{\mu / p^{3}} \frac{\cos i}{\sqrt{1-\sin ^{2} i \sin ^{2}(\omega+f)}} \times \\
(1+e \cos f)^{2}-2 \frac{\mu}{p^{3}} \frac{\sin ^{3} i \cos ^{2}(\omega+f) \sin (\omega+f)}{1-\sin ^{2} i \cos ^{2}(\omega+f)}(1+e \cos f)^{4}
\end{array}\right] .
\end{aligned}
$$

It is assumed that the spacecraft is equipped with a charged surface (screen) of area $S$, with electric charge $q=\int_{S} \sigma d S$ distributed over the surface, with density $\sigma$. Therefore, as in [1], we can write the torque from these forces, relative to the spacecraft's center of mass, as follows:

$$
\vec{T}_{L}=\int_{S} \sigma \vec{\rho} \times(\vec{V} \times \vec{B}) d S
$$

where, $\vec{\rho}$ is the radius vector of the screen's element $d S$ relative to the spacecraft's center of mass, and $\vec{V}$ is the velocity of the element $d S$ relative to the geomagnetic field. Finally, the torque $\vec{T}_{L}$ can be written as follows:

$$
\vec{T}_{L}=\left(T_{L x}, T_{L y}, T_{L z}\right)=q \vec{\rho}_{0} \times A^{T}\left(\vec{V}_{r e l} \times \vec{B}_{o}\right),
$$

or,

$$
\begin{aligned}
& \vec{T}_{L}=\left(T_{L x}, T_{L y}, T_{L z}\right)=\rho_{0} \times A^{T}\left(R_{L}, T_{L}, N_{L}\right)^{T}, \\
& \vec{\rho}_{0}=x_{0} i_{b}+y_{0} j_{b}+z_{0} k_{b}=q^{-1} \int_{S} \sigma \rho d S .
\end{aligned}
$$

$\vec{\rho}_{0}$ is the radius vector of the charged center of the spacecraft relative to its center of mass, and $A^{T}$ is the transpose of the matrix $A$ of the direction cosines $\vec{\alpha}, \vec{\beta}, \vec{\gamma}$.

\section{Analysis of the attitude motion}

The Euler-Poisson equations are used to describe the attitude dynamics of a rigid spacecraft.

$$
\begin{aligned}
& \dot{\vec{\omega}} I+\vec{\omega} \times \vec{\omega} I=\vec{T}_{G}+\vec{T}_{L}, \\
& \dot{\vec{\alpha}}+\vec{\alpha} \times \vec{\omega}=-\Omega \vec{\gamma}, \dot{\vec{\beta}}+\vec{\beta} \times \vec{\omega}=0, \dot{\vec{\gamma}}+\vec{\gamma} \times \vec{\omega}=\Omega \vec{\alpha},
\end{aligned}
$$

where, $T_{G}=3 \Omega^{2} \vec{\gamma} \times \vec{\gamma} I$ is the well known formula of the gravity gradient torque, $I=\operatorname{diag}(A, B, C)$ is the inertia matrix of the spacecraft, $\Omega$ is the orbital angular velocity, and $\vec{\omega}$ is the angular velocity vector of the spacecraft. According to [16], the angular velocity of the spacecraft in the inertial reference frame is $\vec{\omega}=(p, q, r)$, where:

$$
\begin{aligned}
& p=\dot{\psi} \sin \theta \sin \phi+\dot{\theta} \cos \phi \\
& q=\dot{\psi} \sin \theta \cos \phi-\dot{\theta} \sin \phi, \\
& r=\dot{\psi} \cos \theta+\dot{\phi} .
\end{aligned}
$$

\subsection{Attitude motion in the pitch direction}

Assume the attitude motion of the charged spacecraft in the pitch direction, i.e. $\psi=\phi=0, \theta \neq 0$. Applying this condition in the Euler equation of the attitude motion of the spacecraft in Eq (28), we derive the second order differential equation of the motion in the pitch direction:

$A \frac{d^{2} \theta}{d t^{2}}=(C-B)\left(3 \Omega^{2}-1\right) \sin \theta \cos \theta+\left(z_{0} N_{L}-y_{0} T_{L}\right) \sin \theta+\left(y_{0} N_{L}-z_{0} T_{L}\right) \cos \theta$.

$$
\begin{aligned}
& \text { Let } \\
& y_{0}=k z_{0},
\end{aligned}
$$

where, $k$ is arbitrary number. Here $k=1$, corresponds to the spherical shape of satellite, $k>1$ corresponds to complex shapes, and $k<1$ corresponds to a cylindrical shape satellite. Then, equation (31) takes the following form:

$A \frac{d^{2} \theta}{d t^{2}}=(C-B)\left(3 \Omega^{2}-1\right) \sin \theta \cos \theta+z_{0}\left(N_{L}-k T_{L}\right) \sin \theta+z_{0}\left(k N_{L}-T_{L}\right) \cos \theta$.

In the right hand side of this equation, the first term represents the gravity gradient torque, while the second and third terms represent the Lorentz torque.

\subsubsection{Numerical simulations in the pitch direction}

In this section, the equilibrium solutions for the spacecraft will be studied in the pitch direction, using two specific examples. The stability of the derived equilibrium solutions will be discussed using the phase diagrams. By putting the values of orbital elements $a=6900 \mathrm{~km}, e=0001, \mathrm{i}=51^{\circ}$, $f=60^{\circ}$ and $\Omega=1.1 \times 10^{-3} \mathrm{rad} / \mathrm{sec}, B=55 \mathrm{~kg} / \mathrm{m}^{2}, C=57 \mathrm{~kg} / \mathrm{m}^{2}$ and $B_{0}=-8.00 \times 10^{-15} \mathrm{Wbm}$ in equation (33), we get the following equation:

$A \frac{d^{2} \theta}{d t^{2}}=\lambda z_{0}(\cos \theta(0.006+0.011 k)+\sin \theta(0.006 k+0.011))-0.999 \sin 2 \theta$.

where, $\lambda=\frac{q}{m} \mathrm{C} / \mathrm{kg}$ is the charge-to-mass ratio. For a very small value of $\lambda$ and $0 \leq k, z_{0} \leq 3$ the equilibrium points are close to $n \pi / 2$ (Figure 2 (a)). Equilibrium points close to $n \pi$ are stable (black dots in Figure 2), and equilibrium points 
close to $\frac{2 n+1}{2} \pi$ are unstable (red dots in Figure 2). Two typical examples are given for $\lambda=0.001 \mathrm{C} / \mathrm{kg}, k=1, z_{0}=1 \mathrm{~m}$ and $\lambda=1, k=z_{0}=2$. In the first case, the equilibrium points are at $\theta=0, \frac{\pi}{2}, \pi, \frac{3 \pi}{2}, 2 \pi$; and in the second case, the equilibrium points are at $\theta=0.013,1.55,3.12,4.69,6.26$. They are shown in Figure 2 (a) and Figure 2 (b), respectively, which look almost identical. For higher or negative values of $\lambda$ (charge to mass ratio), or higher values of $z_{0}$, the position and stability of the equilibrium points change significantly, see Figures 3 to 5 . In Figure 3 (left), there are two equilibrium positions at $\theta$ $=2.68 \mathrm{rad}$ (stable) and $\theta=5.03$ (unstable), for $\lambda=5$ and $z_{0}=7$. In Figure 3 (right), there are four equilibrium positions at $\theta=1.75 \mathrm{rad}$ (unstable), 3.54rad (stable), 4.37 rad (unstable), $6.04 \mathrm{rad}$ (stable), for, $\lambda=-4$ and $z_{0}=4$. A comparison of Figure 2 and Figure 3 clearly shows the effect of changing values of $\lambda$ and $z_{0}$, as both the position and stability of equilibrium positions are affected.

To further analyze the effects of parameters $\lambda$ and $k$ on the existence and stability of equilibrium points, we take another example, with $B=240 \mathrm{~kg} / \mathrm{m}^{2}$ and $C=250 \mathrm{~kg} / \mathrm{m}^{2}$. In this case, the differential equation in the pitch direction becomes:

$A \frac{\mathrm{d}^{2} \theta}{d t^{2}}=\cos \theta\left((0.006+0.011 k) \lambda \mathrm{z}_{0}-10 \sin \theta\right)+(0.01+0.006 k) \lambda \mathrm{z}_{0} \sin \theta$

In this case for $0 \leq \theta \leq 2 \pi$, there are five equilibrium points at $\theta \approx 0, \frac{\pi}{2}, \pi, \frac{3 \pi}{2}, 2 \pi$, when $-5 \leq \lambda \leq 5$. In the case when $B>C$ and $\lambda=0.001$, refer Figure 4 (left), the equilibrium positions at $\theta=0, \pi, 2 \pi$ are unstable, and at $\theta \approx \frac{\pi}{2}, \frac{3 \pi}{2}$ are stable; while in the case $B<C$ and $\lambda=1$, refer Figure 4 (centre), the equilibrium positions at $\theta=0, \pi, 2 \pi$ are stable, and at $\theta \approx \frac{\pi}{2}, \frac{3 \pi}{2}$ are
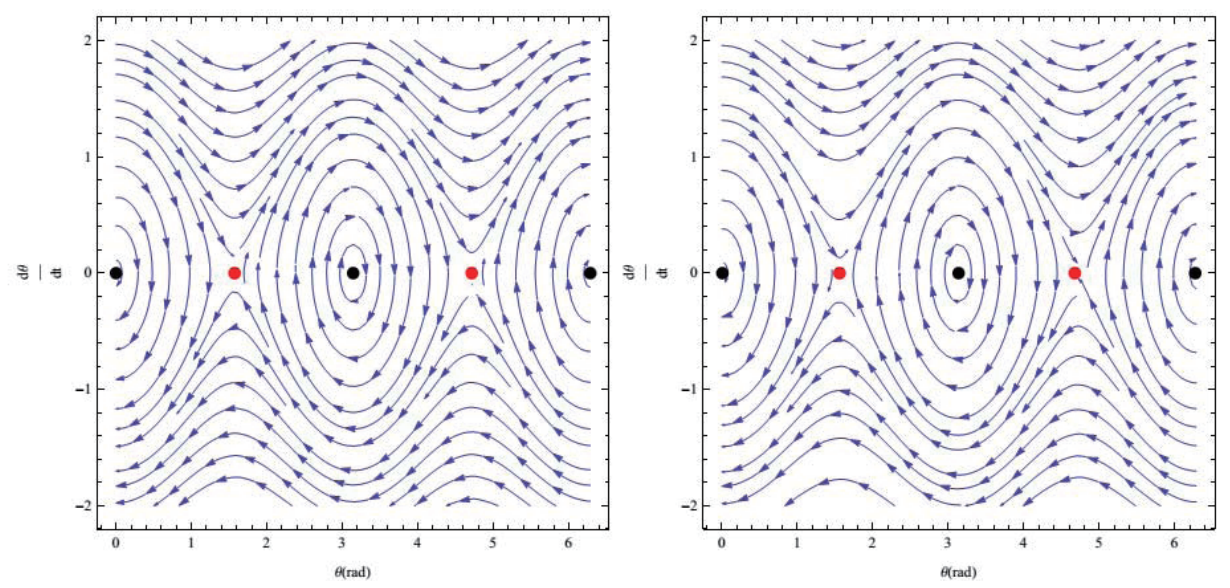

Fig. 2. Trajectory in the $\theta-\frac{d \theta}{d t}$ phase plane, when $a=6900 \mathrm{~km}, e=0001, i=51^{\circ}, f=60^{\circ}\left(\right.$ Left): $\lambda=0.001, k=z_{0}=1, A=53, B=55, C=57$. (Right): $\lambda=1, k=z_{0}=2$, $A=53, B=55, C=57$. Black dots correspond to stable equilibrium points, and red dots correspond to unstable equilibrium points.
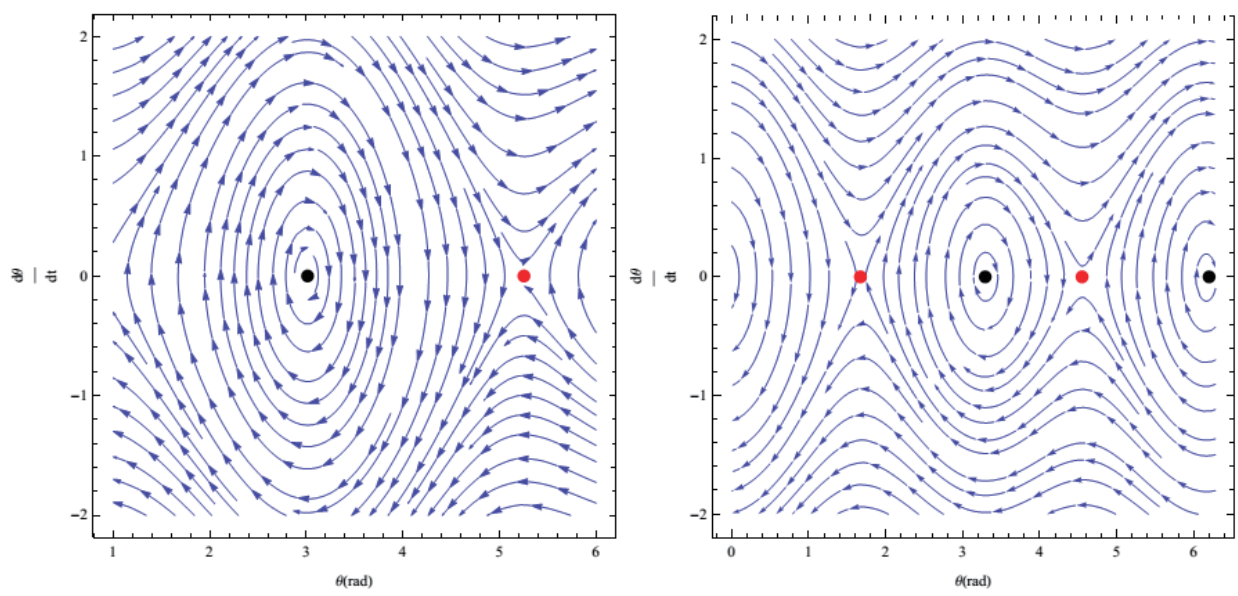

Fig. 3. Trajectory in the $\theta-\frac{d \theta}{d t}$ phase plane, when $a=6900 \mathrm{~km}, e=0001, i=51^{\circ}, f=60^{\circ}$ (Left): $\lambda=5, k=3, z_{0}=7, A=53, B=55, C=57$. (Right): $\lambda=-4, k=3, z_{0}=4$, $A=53, B=55, C=57$. Black dots correspond to stable equilibrium points, and red dots correspond to unstable equilibrium points. 

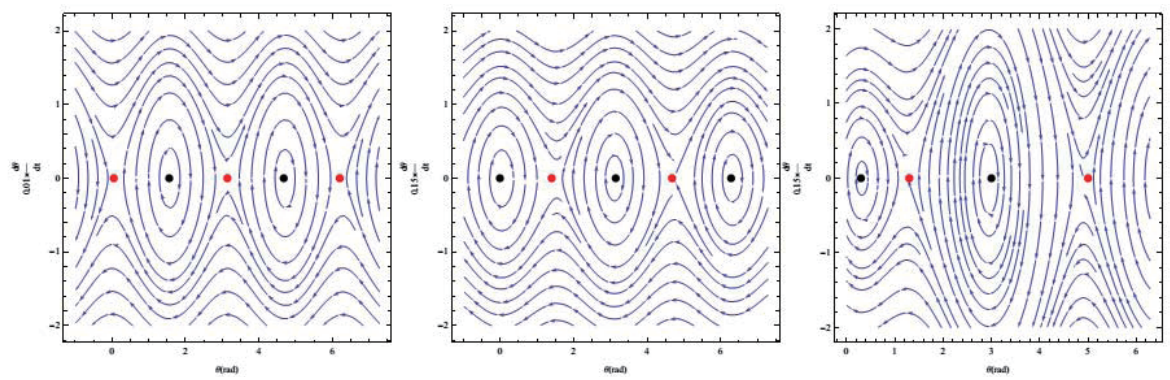

Fig. 4. Trajectory in the $\theta-\frac{d \theta}{d t}$ phase plane, when $a=6900 \mathrm{~km}, e=0001, i=51^{\circ}, f=60^{\circ}$ (Left): $\lambda=0.001, k=1, z_{0}=1, A=53, B=250, C=240$. (Center): $\lambda=1, k=1$, $z_{0}=1, B=240, C=250$. (Right): $\lambda=20, k=1, z_{0}=7, B=240, C=250$. Black dots correspond to stable equilibrium points, and red dots correspond to unstable equilibrium points.

unstable. For higher values of $\lambda$, the equilibrium position at $\theta=2 \pi$ disapears, as can be seen in Figure 4 (right), which is given for $\lambda=20$. If we compare Figures 2,3 and 4 , for changing values of $\lambda$ and $z_{0}$, the equilibrium positions moved to the right. Therefore we can conclude that the values of $\lambda$ and $z_{0}$ have a significant effect on the location and stability of the equilibrium points. Hence, these parameters can be used for passive control. A complete set of equilibrium positions is given in Figure 5, to show the effect of the components of moment of inertia ( $B$ and $C$ ) of the spacecraft on the location and existence of equilibrium positions. Figure 5 (left) is given for $B=55$ and $C=57$, where the equilibrium positions have a complicated dependence on the values of $\lambda$ and $k$. Figure 5 (left) is given for $B=240$ and $C=250$, where the equilibrium positions have an almost linear dependence on $\lambda$ and $k$.

\subsection{Attitude motion in the roll direction}

In this section, we study the attitude motion of the charged spacecraft in the roll direction, i.e. $\psi=\theta=0, \phi \neq 0$. Applying this condition to the Euler equation of the attitude motion of the spacecraft in Eq (28), we obtain the second order differential equation of the motion in the roll direction.

$C \frac{d^{2} \phi}{d t^{2}}=\Omega^{2}(A-B) \sin \phi \cos \phi-\left(x_{0} R_{L}-y_{0} T_{L}\right) \sin \phi+\left(x_{0} T_{L}-y_{0} R_{L}\right) \cos \phi$.

Let

$y_{0}=k x_{0}$.

Then, equation (36) takes the form:

$C \frac{d^{2} \phi}{d t^{2}}=\Omega^{2}(A-B) \sin \phi \cos \phi+x_{0}\left(k T_{L}-R_{L}\right) \sin \phi+x_{0}\left(T_{L}-k R_{L}\right) \cos \phi$

In the right hand side of this equation, the first term represents the gravity gradient torque, and the second and third terms represent the Lorentz torque.

\subsubsection{Numerical simulations in the roll direction}

In this section, the equilibrium solutions for the spacecraft will be studied in the roll direction, using two specific examples, in the same way as has been done for the pitch direction. This will allow us to make comparisons, wherever possible. The stability of the derived equilibrium solutions will be discussed, using the phase diagrams. Let $\Omega=1.1 \times 10^{-}$
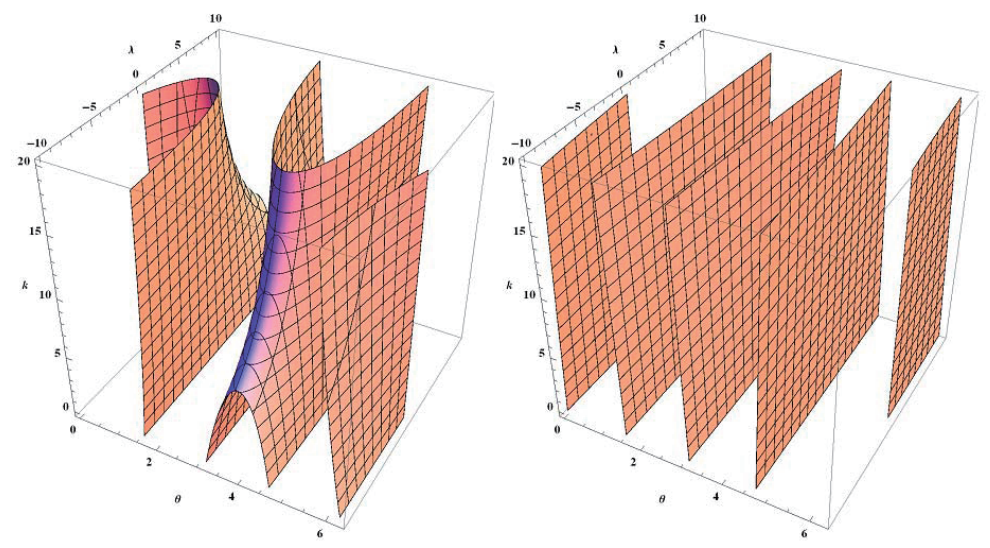

Fig. 5. Family of equilibrium solutions in the pitch direction when $z_{0}=1, a=6900 \mathrm{~km}, e=0001, i=51^{\circ}, f=60^{\circ}$ (Left) $B=55, C=57$, (Right) $B=250, C=240$. 
${ }^{3} \mathrm{rad} / \mathrm{sec}, \quad B_{0}=-8 \times 10^{-15} \mathrm{Wbm}, \quad A=53 \mathrm{~kg} / \mathrm{m}^{2}$ and $B=55 \mathrm{~kg} / \mathrm{m}^{2}$. Then, the differential equation in the roll direction becomes:

$$
C \frac{d^{2} \phi}{d t^{2}}=\left[3.49 \times 10^{-4} \lambda(\sin \phi+\cos \phi)-1.21 \times 10^{-6} \sin 2 \phi\right]
$$

where, $\lambda=\frac{q}{m} C / \mathrm{kg}$ is the charge-to-mass ratio. It is straightforward to show that for each value of $\phi$, there exists at least one $\lambda$ at which $\frac{d^{2} \phi}{d t^{2}}=0$. Therefore, to have an equilibrium, the following relationship between $\lambda$ and $\phi$ must hold, which shows the existence of a continuous family of equilibrium solutions from 0 to $2 \pi$, except at $\phi=2.356$ and $\phi=5.497$ :

$$
\lambda=\frac{3.5 \times 10^{-3} \sin (2 \phi)}{\cos \phi+\sin \phi} .
$$

Conversely, there exists at least two equilibrium points for each value of $\lambda$. One of these equilibrium points is stable, and one of them is unstable. In the special case of $\lambda$ $=0$, which corresponds to a satellite without charge, there are five equilibrium points at $\phi=0, \frac{\pi}{2}, \pi, \frac{3 \pi}{2}$ and $2 \pi$. The equilibrium points $0, \pi$ and $2 \pi$ are stable, and the remaining two are unstable. They are shown in Figure 6 (left). In the cases of non-zero $\lambda$ i.e. charged spacecraft, there are only two equilibrium positions where one of them is stable, and another one is unstable. Two typical examples are shown in Figure 6 (centre) and Figure 6 (right), for $\lambda=-10 \mathrm{C} / \mathrm{kg}$ and $\lambda=10 \mathrm{C} / \mathrm{kg}$, respectively. The numerical value of $\lambda$ has a significant effect on the location or nature of the equilibrium point. For $\lambda>0$, the two equilibrium points are found at around $\phi=2.28 \mathrm{rad}($ stable) and $\phi=5.57 \mathrm{rad}($ unstable). For $\lambda<0$, the locations of equilibrium points remain the same, while their nature is reversed.

To further analyze the effect of the parameters $\lambda$ on the existence and stability of equilibrium points, we take another example with $A=230$ and $B=240$. In this case, the equation of motion in the roll direction becomes:

$$
C \frac{d^{2} \phi}{d t^{2}}=\left[3.49 \times 10^{-4} \lambda(\sin \phi+\cos \phi)-6.05 \times 10^{-6} \sin (2 \phi)\right]
$$

As in the first case, to have an equilibrium, the following relationship between $\lambda$ and $\phi$ must hold, which shows the existence of a continuous family of equilibrium solutions from 0 to $2 \pi$, except at $\phi=2.356$ and $\phi=5.497$.

$$
\lambda=\frac{0.017 \sin (2 \phi)}{(\sin \phi+\cos \phi)} .
$$

In this case, the locations of the equlibrium points remain identical to the case discussed above. The nature of the orbits is reversed, except for the case when $\lambda=0$.

\subsection{Attitude motion in the yaw direction}

In this section, the attitude motion of the charged spacecraft in the yaw direction will be discussed. Let $\phi=\theta=0, \psi \neq 0$. Applying this condition to the Euler equation of the attitude motion of the spacecraft in Eq (28), the equation of the motion in the yaw direction is obtained.

$$
(A-B-C) \Omega \sin \psi \frac{d \psi}{d t}=z_{0}\left[R_{L} \cos \psi-T_{L} \sin \psi\right]-x_{0} N_{L}
$$

Let

$x_{0}=k z_{0}$.

Then, equation (43) takes the following form.

$$
\frac{d \psi}{d t}=\frac{z_{0}}{(A-B-C) \Omega}\left[R_{L} \cot \psi-T_{L}-k \frac{N_{L}}{\sin \psi}\right] .
$$

\subsubsection{Numerical simulations for attitude motion in the yaw direction}

It is clear from the equation of motion in the yaw direction that the values of $A, B, C$ and $z_{0}$ have no effect on the
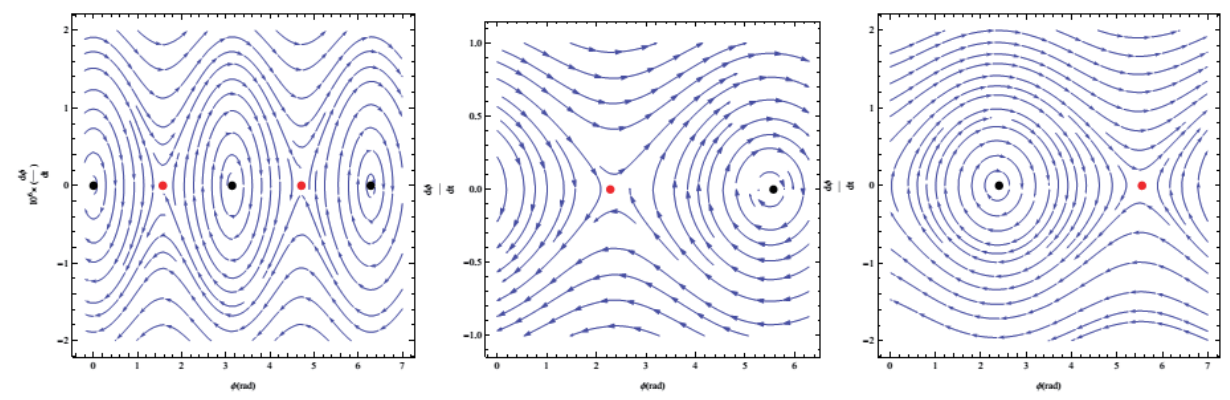

Fig. 6. Trajectory in the $\theta-\frac{d \theta}{d t}$ phase plane, when $a=6900 \mathrm{~km}, e=0001, i=51^{\circ}, f=60^{\circ}$ (Left): $\lambda=0, A=53, B=55$. (Center): $\lambda=-10, A=53, B=55$ (Right): $\lambda=10$, $A=53, B=55$. Black dots correspond to stable equilibrium points, and red dots correspond to unstable equilibrium points. 
existence of equilibrium solutions. For any $\psi=\psi_{0}$ to be an equilibrium, it must satisfy the following equation:

$$
R_{L} \cos \psi-T_{L} \sin \psi-k N_{L}=0
$$

Let $z_{0}=1, \quad a=6900 \mathrm{~km}, \quad e=0.001, \quad i=51^{\circ}$, and $f=60^{\circ}$; then, equation (46) takes the following form:

$$
\lambda(0.006-0.006 \cot \psi-0.011 k \csc \psi)=0 \text {. }
$$

It is obvious from equation (47) that $\lambda$ has no effect on the existence of equilibrium positions. For each $k \in(0$, $0.6126)$, there is one equilibrium point; and for $k \in(0.6126$, 0.8435), there are two equilibrium points. For all other values of $k$, there are no equilibrium points. For a complete list of these points, see Figure 7 , which shows the above mentioned intervals for the existence of one and two equilibrium positions. Figure 7 also confirms that there are no equilibrium positions for $k>0.8$.

Using the same procedure used for the roll and pitch directions, it can easily be shown that the equilibrium positions that exist when $0<k<0.6126$ is a source node or unstable node. Similiarly, one of the two equilibria that exist when $0.6126<k<0.8435$ is a source node, and the second

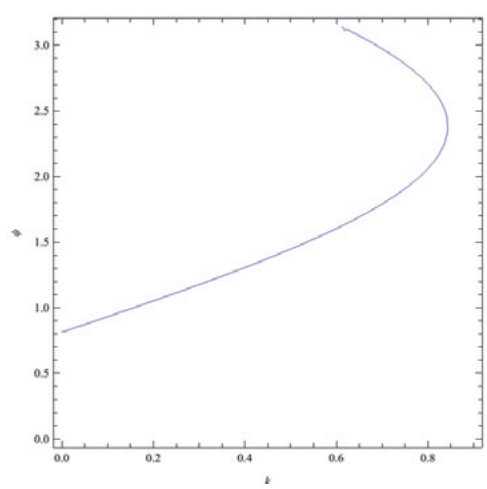

Fig. 7. Complete list of equilibrium solutions in the yaw direction, when $z_{0}=1, a=6900 \mathrm{~km}, e=0.001, i=51^{\circ}$, and $f=60^{\circ}$
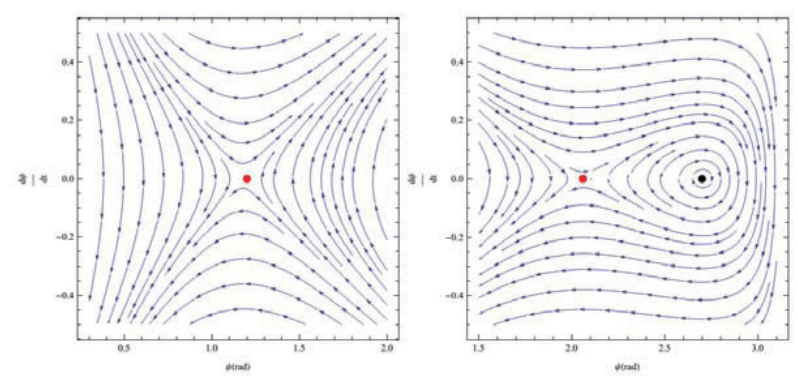

Fig. 8. Trajectory in the $\psi-\frac{d \psi}{d t}$ phase plane, when $z_{0}=1, a=6900 \mathrm{~km}$, $e=0.001, i=51^{\circ}, f=60^{\circ}$ (Left): $k=0.3$. (Right): $k=0.8$. Black dots correspond to stable equilibrium points, and red dots correspond to unstable equilibrium points. one is a stable centre. An example is given in Figure 8, for fixed values of $k=0.3$ and $k=0.8$. When $k=0.3$, Figure 8 (left), there is only one equilibrium position at $\psi=1.17 \mathrm{rad}$, which is unstable; and at $k=0.8$, Figure 8 (right), there are two equilibrium positions, at $\psi=2.076 \mathrm{rad}$, and $\psi=2.70 \mathrm{rad}$. The first equilibrium is unstable, while the second one is stable. As opposed to the other two directions, the only parameter that affects the existence of equilibrium positions is $k$, which is connected to components of the radius vector $\left(\vec{\rho}_{0}\right)$ of the charged center of the spacecraft, relative to the center of mass of the spacecraft.

\section{Conclusions}

In this paper, a new approach for the torque due to the Lorentz force as a function of the orbital elements of a spacecraft is developed, for a general shape of a charged spacecraft moving in an elliptic orbit. The equations of motion for the attitude orientation are developed, using Euler-Poisson Equations. We investigated the existence of equilibrium positions, and its stability separately in pitch, roll and yaw directions. The Lorentz force was introduced as the source for attitude stablization of a general shape spacecraft. We identified attitude stablization parameters for each direction (pitch, roll and yaw), and determined specific values of the aforementioned parameters. The numerical results show the exact location of stable and unstable equilibrium positions. It is shown through numerical simulations that the charge-to-mass ratio, and the radius vector $\left(\vec{\rho}_{0}\right)$ of the charged center of the spacecraft relative to the center of mass of the spacecraft, work as a passive control, in the pitch and roll directions. The charge-tomass ratio has no role to play in either the existence, or the stability, of equilibrium positions in the yaw direction. The only parameter of stability in the yaw direction is $\vec{\rho}_{0}$. It is also shown that there will be no equilibrium solution, if the $x$-component of $\vec{\rho}_{0}$ is greater than $z_{0}$. Therefore, monitoring the level of charge on the spacecrafts' surface is important, in the sense that it can be used as passive control for the attitude dynamics of the spacecraft. It is not possible to provide an exact range for $\lambda$, but a range of -1 to 1 will be the easiest to achieve. Higher values of $\lambda$ are theoretically possible, and can be realised in the case of very large satellites.

\section{References}

[1] Tikhonov, A. A., Spasic, D. T., Antipov, K. A. and Sablina, M. V., “Optimizing theelectrodynamical stabilization method 
for a man-made Earth satellite", Automation and Remote Control, Vol. 72, No. 9, 2011, pp. 1898-1905.

[2] Pollock, G. E., Gangestad, J. W., and Longuski, J. M., "Analytical solutions for the relative motion of spacecraft subject to Lorentz-force perturbations", Acta Astronautica, Vol 68, No. 1, 2011, pp. 204-217.

[3] Chao, P. and Yang, G., "Lorentz force perturbed orbits with application to J2-invariant formation", Acta Astronautica, Vol . 77, No. 1, 21012, pp. 12-28.

[4] Griffiths, D. J., Introduction to Electrodynamics, Prentice Hall, Englewood Cliffs, New Jersey, 1989.

[5] Abdel-Aziz, Y. A., "Lorentz force effects on the orbit of a charged artificial satellite: A new approach", Applied Mathematical Sciencs, Vol. 1, No. 31, 2007, pp. 1511-1518.

[6] Vokrouhlický, D., "The geomagnetic effects on the motion of an electrically charged artificial satellite", Celestial Mechanics and Dynamical Astronomy, Vol. 46, No. 1, 1989, pp. 85-104.

[7] Gangestad, J. W., Pollock, G. E and Longuski, J. M., "Lagrange's planetray equations for the motion of electrostatically charged spacecraft", Celest Mech Dyn. Astr., Vol. 108, No. 2, 2010, pp. 125-145.

[8] Peck, M. A., "Prospects and challenges for Lorentz augmented orbits" AIAA Guidance, Navigation, and Control Conference, AIAA, San Francisco, CA, 2005, pp. 5995

[9] King, L. B., Parker, G.G., Deshmukh, S. and Chong, J.H., "Study of interspace coulomb forces and implications for formation flying, Journal of Propulsion and Power", Vol. 19, No. 3, 2003, pp. 497-505.

[10] Natarajan, A. and Schaub, H., "Linear dynamics and stability analysis of a coulomb tether formation", Journal of Guidance Control and Dynamics, Vol. 29, 2006, pp. 831-839.

[11] Streetman, B., and Peck, M. A., "New synchronous orbits using the geomagnetic Lorentz force", Journal of Guidance Control and Dynamics, Vol. 30, No 6, 2007, pp. 1677-1690.

[12] Utako, Y. and Hiroshi, Y., “Two-craft coulomb-force formation dynamics and stability analysis with debyelength characteristics", AIAA/AAS Astrodynamics Specialist Conference, AIAA, Honolulu, Hawaii, 2008, pp.7361

[13] Hiroshi, Y., Katsuyuki, Y and Mai, B., Spacecraft formation dynamics under the influence of geomagnetic Lorentz force, Twenty-seventh International Symposium on Space Technology and Science, 2009.

[14] Abdel-Aziz, Y. A., "Attitude stabilization of a rigid spacecraft in the geomagnetic field", Adv. Space Res, Vol. 40, No 1, 2007, pp. 18-24.

[15] Yamakawa, H., Hachiyama, S and Bando, M., "Attitude dynamics of a pendulum-shaped charged satellite",. Acta Astronautica, Vol. 70, 2012, pp. 77-84.

[16] Wertz, J. R., Spacecraft attitude determination and control, D. Reidel Publishing Company, Dordecht, Holland,1978. 